\title{
Charge renormalization of charged spheres based on thermodynamic properties
}

\author{
Wei Lun Hsin and Tzu-Yu Wang \\ Department of Chemical and Materials Engineering, National Central University, Jhongli, Taiwan 320, \\ Republic of China \\ Yu-Jane Sheng \\ Department of Chemical Engineering, National Taiwan University, Taipei, Taiwan 106, Republic of China \\ Heng-Kwong Tsao ${ }^{\text {a) }}$ \\ Department of Chemical and Materials Engineering, National Central University, Jhongli, Taiwan 320, \\ Republic of China
}

(Received 9 April 2004; accepted 21 June 2004)

\begin{abstract}
At strong electrostatic coupling, counterions are accumulated in the vicinity of the surface of the charged particle with intrinsic charge $Z$. In order to explain the behavior of highly charged particles, effective charge $Z^{*}$ is therefore invoked in the models based on Debye-Hückel approximation, such as the Derjaguin-Landau-Verwey-Overbeek potential. For a salt-free colloidal suspension, we perform Monte Carlo simulations to obtain various thermodynamic properties $\omega$ in a spherical Wigner-Seitz cell. The effect of dielectric discontinuity is examined. We show that at the same particle volume fraction, counterions around a highly charged sphere with $Z$ may display the same value of $\omega$ as those around a weakly charged sphere with $Z^{*}$, i.e., $\omega(Z)=\omega\left(Z^{*}\right)$. There exists a maximally attainable value of $\omega$ at which $Z=Z^{*}$. Defining $Z^{*}$ as the effective charge, we find that the effective charge passes through a maximum and declines again due to ion-ion correlation as the number of counterions is increased. The effective charge is even smaller if one adopts the Debye-Hückel expression $\omega_{\mathrm{DH}}$. Our results suggest that charge renormalization can be performed by chemical potential, which may be observed in osmotic pressure measurements. (C) 2004 American Institute of Physics. [DOI: 10.1063/1.1782431]
\end{abstract}

\section{INTRODUCTION}

The concept of the effective charge is commonly used in the literature to describe the equilibrium and dynamic properties of colloidal solutions. The basic idea of charge renormalization is that owing to strong electrostatic coupling, counterions accumulate in the vicinity of the surface of the particle with intrinsic charge $Z$. As a result, the decorated object (charged particle plus counterions) may be regarded as a single entity which possesses an effective charge, $Z^{*} .^{1,2}$ The effective charge (in absolute value) can be much smaller than the intrinsic charge $Z^{*} \ll Z$. The determination of the effective charge depends on which property is considered. This quantity is often regarded as an adjustable parameter in a fit of experimental data with approximated models. For example, the effective charge can be inferred from electrophoresis or voltammetry. ${ }^{3}$

The accumulation of counterions close to the charged particle, so-called counterion condensation, is the sufficient condition of charge renormalization. Evidently, the intrinsic charge carried by the particle $Z$ and the particle size $a$ are important factors. However, whether counterion accumulation occurs at the surface of an isolated charged object depends on the geometric shape as well. At infinite dilution, counterion condensation takes place only at infinitely ex-

${ }^{a}$ Electronic mail: hktsao@cc.ncu.edu.tw tended planes and cylinders, but not at spherical particles. This can be illustrated by considering the electric potential felt by a single counterion at the particle surface. For a charged plate it grows linearly with the size of the plate, $\psi_{s} \sim-a$. Thus the infinitely extended plate possesses infinite surface potential and is able to bind any counterions. The renormalized charge per unit area is effectively zero. This is completely inverted for an isolated sphere because the potential energy of a counterion on the surface of a totally ionized sphere, $\psi_{s} \sim-Z a^{-1}$, is finite. The entropy associated with the counterion is proportional to $k_{B} T \ln V$ with the available volume $V \rightarrow \infty$ at infinite dilution. Therefore, the charged sphere is unable to bind a counterion at finite temperature. In the intermediate case of a rodlike particle, one has $\psi_{s}$ $\sim-\ln a$. For an infinitely extended line, the electrostatic energy gain is able to balance the counterion entropy loss. Manning ${ }^{4}$ and Oosawa ${ }^{5}$ therefore pointed out that the line charge density of a polyelectrolyte is limited to a maximum value given by this balance point of energy and entropy. All other counterions are "condensed" to the polyelectrolyte.

Despite the fact that charge renormalization does not occur for an isolated charged sphere, ${ }^{6}$ the counterion entropy, $\sim-k_{B} T \ln c$, is finite in all practical salt-free colloidal systems due to finite counterion concentration $c$. A salt-free colloidal dispersion contains electrically charged particles with counterions dissociated from the particle's surface. A typical example is charged micelles, which are formed by ionic sur- 
factant such as $n$-dodecyle sulfate, surrounded by counterions. Charge renormalization ${ }^{7}$ essentially corresponds to a battle fought between energy and entropy in minimizing the free energy of a solution of mobile charges near charged particles. As a consequence, one anticipates that charge renormalization takes place eventually when the electrostatic energy gain overwhelms the counterion entropy loss.

Since a colloidal dispersion, involving many charged particles and small ions, is a very complicated system, one commonly adopts a spherical Wigner-Seitz (WS) cell to investigate the physical properties associated with colloids. ${ }^{1-4,8-11}$ The cell model approximation reduces the theoretical description of the whole system to just one cell. While the interactions among charged particles are neglected, the interaction between small ions with "their" charged particle as well as with small ions are explicitly taken into account in the same cell. Therefore, the cell model approach can be regarded as an approximate attempt to factorize the partition function in the particle coordinates and hence the many-colloid problem is replaced by a singlecolloid problem. ${ }^{12}$ The symmetry of the cell may reduce the problem further to a one-dimensional one and allows an analytic treatment. The radius of the WS cell, $R$, is often related to the volume fraction of charged particles, $\phi$, by $R$ $=a \phi^{-1 / 3}$.

On the basis of the spherical WS cell, the effective charge can be determined theoretically according to various definitions. The simple and intuitive definition is the twostate approximation, i.e., condensed and free counterions. The boundary between the two states is defined arbitrarily. For example, the position at which the local concentration of counterions equals the mean value is chosen so that the counterions between the particle surface and the boundary neutralize an equivalent number of charges on the particle. The effective charge is therefore the rest of the charge carried by the particle. ${ }^{3}$ Similarly, one can also define the boundary as the position at which the interaction energy of the counterion with the parent particle is equal to the thermal energy. ${ }^{13}$ Nevertheless, such definitions of $Z^{*}$ do not relate to thermodynamic quantities.

Another approach of charge renormalization is the electric field felt by a counterion far from the parent particle. ${ }^{1}$ The electrostatic stabilization of colloidal dispersions against aggregation is usually depicted by the DLVO theory, named after Derjaguin, Landau, Verwey, and Overbeek. ${ }^{14}$ The electrostatic repulsion in the DLVO theory originates from the effective interaction potential between two charged colloids based on the Poisson-Boltzmann (PB) mean-field description of the ion clouds. Within a linearized approximation, it takes the well-known Debye-Hückel (DH) form, $u(r) \sim Z^{2} \exp$ $(-\kappa r) / r$, where $Z$ is the intrinsic charge of the particle and $\kappa$ represents the inverse Debye screening length. This approximation becomes inadequate to describe highly charged particles because the electrostatic energy of counterions near the particle's surface exceeds the thermal energy $k_{B} T$. Far from the charged particle, nevertheless, the electrostatic potential still follows the DH form because the thermal energy becomes dominant. Although the intrinsic surface charge of a particle $Z$ can be directly measured, the DLVO theory often requires an effective charge $Z^{*}$, which accounts for screening of the intrinsic charge by counterions. Within the framework of the effective pair potential, the effective charge can be determined by matching the DH form to the long distance behavior of the "exact" PB solution. ${ }^{1}$

In the cell model, the thermodynamic properties of a colloidal dispersion, such as osmotic pressure $\pi(\phi, Z, a)$ and bulk osmotic modulus, are related to the counterion concentration on the surface of the WS sphere with radius $R, c(R) .{ }^{1}$ If the effective charge is known, one is able to evaluate $c\left(R ; Z^{*}\right)$ by solving the linearized $\mathrm{PB}$ equation analytically and hence the osmotic pressure can be predicted, $\pi\left(R ; Z^{*}\right)$. Following the opposite line of reasoning, the measurement of a particular thermodynamic property $\omega(Z)$ of a colloidal dispersion can yield the effective charge through the theoretical value of $\omega\left(Z^{*}\right)$ calculated from the linearized PB theory based on the cell model. In other words, the effective charge determined by the second approach is in a close relation with a thermodynamic quantity. A similar framework can also be established for the determination of the effective charge from transport properties of a colloidal dispersion, such as selfdiffusion coefficient ${ }^{11}$ and electrophoretic mobility ( $\zeta$ potential).

Previous studies disclose the fact that charge renormalization takes place for highly charged particles. Due to strong screening, the effective charge concept is invoked for explaining the far-field behavior of a highly charged particle by the one associated with a weakly charged particle. Based on the PB theory, it is generally accepted that there is a maximum for $c(R)$ as $Z$ diverges. In other words, at a given volume fraction, the effective charge of a spherical colloid reaches a plateau value for large intrinsic charge because no more counterions are able to desorb from the particle's surface. ${ }^{1,8}$ The thermal energy of a counterion is exactly compensated by the reversible work required to remove a counterion from the surface of the particle. However, some simulation studies ${ }^{9}$ showed that the effective charge of a spherical colloid does not reach a plateau value for large intrinsic charge. Instead, the effective charge passes through a maximum, and decreases again as the number of counterions increases.

In this paper we investigate the charge renormalization of a colloidal dispersion by employing the WS cell. In order to provide the effective charge with proper thermodynamic meaning, the charge renormalization is performed based on the thermodynamic properties of the system, such as chemical potential or osmotic pressure. In Sec. II we analyze the thermodynamic properties of the system derived from the partition function. In the limit of very weak electrostatic coupling, we are able to obtain the electric potential, counterion concentration, and chemical potential without resorting to the PB equation under the DH approximation. In Sec. III the details of Monte Carol (MC) simulations are briefly described. In Sec. IV we show the agreement between the theoretical results and $\mathrm{MC}$ results in the very weak coupling regime. Furthermore, we demonstrate that two colloidal dispersions with the same particle size and volume fraction may display exactly the same thermodynamic property even though they possess very different charges. Therefore, two 
methods based on thermodynamic properties are proposed to determine the effective charge.

\section{THEORY}

Appropriate thermodynamic properties for charge renormalization include osmotic pressure, chemical potential, particle surface potential, and mean-square dipole moment. A natural choice to study charge renormalization by Monte Carlo simulations is based on the osmotic pressure, which is the counterion concentration at the boundary of the cell. However, this quantity is relatively difficult to be measured accurately in the dilute limit. Another possible choice is the chemical potential $\mu$, which is a constant throughout the system at thermodynamic equilibrium. In this paper, we adopt the chemical potential to define the effective charge. Following the conventional treatment, one can always define the effective charge by the Debye-Hückel chemical potential $\mu_{\mathrm{DH}}$, that is, $\mu(Z)=\mu_{\mathrm{DH}}\left(Z^{*}\right)$ with $Z>Z^{*}$. In the following paragraph, we aim to derive the $\mathrm{DH}$ expression associated with the thermodynamic property, such as $\mu_{\mathrm{DH}}$. First of all, we write down the partition function. The relevant properties can then be related to the partition function. The Helmholtz free energy can be divided into two parts. The chemical potential is then obtained with two different contributions. As the intrinsic charge of the colloidal particle is increased, one contribution increases while the other one declines.

On the basis of the WS cell model, we consider a charged particle with radius $a$ and valency $-Z$ located at the center of a spherical cell of radius $R$. There are $N$ counterions with valency $z_{c}$ distributed within the cell. The electroneutrality condition is satisfied because of $Z=N z_{c}$. The partition function of the system is expressed as

$$
\mathcal{Z}\left(a, R, z_{c}, Z, \ell_{B}\right)=\frac{1}{N !} \int \cdots \int e^{-\beta \mathcal{H}} d \mathbf{r}_{1} \cdots d \mathbf{r}_{N},
$$

where $\ell_{B}=e^{2} / 4 \pi \epsilon_{r} \epsilon_{0} k_{B} T(\simeq 0.71 \mathrm{~nm}$ in aqueous solution at $298 \mathrm{~K})$ denotes the Bjerrum length. The Hamiltonian $\mathcal{H}$ is given by

$$
\beta \mathcal{H}=\ell_{B}\left[-\sum_{i=1}^{N} \frac{Z z_{c}}{r_{i}}+\sum_{\substack{i=1 \\ i \neq j}}^{N} \sum_{j=1}^{N} \frac{z_{c}^{2}}{\left|\mathbf{r}_{i}-\mathbf{r}_{j}\right|}\right] .
$$

For simplicity, the dielectric mismatch between the charged particle and the solvent is ignored. The effect of dielectric contrast will be examined later by Monte Carlo simulations. In the dilute limit (in terms of the mean counterion concentration), the finite size effect of counterions can be neglected as well. In fact, the strong Coulomb repulsion between counterions at short separation renders the excluded volume effect. Equation (2) can be made dimensionless in terms of the Bjerrum length associated with counterions with valency $z_{c}(\lambda)$ and the number of counterions $N=Z / z_{c}$,

$$
\beta \mathcal{H}=\left[-\sum_{i=1}^{N} \frac{\left(Z / z_{c}\right)}{\hat{r}_{i}}+\sum_{\substack{i=1 \\ i \neq j}}^{N} \sum_{j=1}^{N} \frac{1}{\left|\hat{\mathbf{r}}_{i}-\hat{\mathbf{r}}_{j}\right|}\right],
$$

where the dimensionless position is $\hat{\mathbf{r}}=\mathbf{r} / \lambda$ with $\lambda=z_{c}^{2} \ell_{B}$. On the right-hand side of Eq. (3), the first term comes from the electrostatic attraction between counterions and the charged particle and is proportional to $N^{2} z_{c}^{2}$. The second term denotes the electrostatic repulsion among counterions and is essentially proportional to $N(N-1) z_{c}^{2} / 2$.

In accordance with the partition function, all thermodynamic properties can be obtained. The Helmholtz free energy is related to the partition function by

$$
\beta \mathcal{F}=-\ln \mathcal{Z}=N\left[\ln \frac{N}{V}-1\right]-\ln \left(1+\Omega_{N}\right),
$$

where

$$
\Omega_{N}[R / \lambda, a / \lambda]=\frac{1}{\hat{V}^{N}} \int \cdots \int\left(e^{-\beta \mathcal{H}}-1\right) d \hat{\mathbf{r}}_{1} \cdots d \hat{\mathbf{r}}_{N} .
$$

The available volume is $V=(4 \pi / 3)\left(R^{3}-a^{3}\right)$. Equations (3) and (5) reveal that the property associated with $\Omega_{N}$ only is a function of $a / \lambda, R / \lambda$, and $Z / z_{c}(=N)$. On the other hand, one is unable to express the total free energy simply as a function of $a / \lambda, R / \lambda$, and $Z / z_{c}$. From the free energy, the chemical potential is given by

$$
\beta \mu=\left(\frac{\partial \mathcal{F}}{\partial N}\right)_{Z, T}=\ln \frac{N}{V}-\frac{\partial}{\partial N} \ln \left[1+\Omega_{N}\left(\frac{R}{\lambda}, \frac{a}{\lambda}\right)\right] .
$$

This result indicates that the chemical potential can be separated into two parts, the ideal chemical potential $\mu_{i}$ $=k_{B} T \ln (N / V)$ and the configuration chemical potential $\mu_{c}\left(\Omega_{N}\right), \mu=\mu_{i}+\mu_{c}$. The former denotes the entropy contribution without electrostatic interactions and grows with the number of counterions. The latter represents the electrostatic contribution and always declines with increasing $Z / z_{c}$. Note that for a given set of $\{a / \lambda, R / \lambda\}, \mu_{c}$ is a constant but $\mu_{i}$ may vary with the combination of $\{a, R, \lambda\}$ because the volume $V$ is independent of $\lambda$.

Similarly, the electric potential is defined as

$$
\begin{aligned}
\beta e \psi(\mathbf{r})= & \frac{1}{N !} \int \cdots \int l_{B}\left[-\frac{Z}{|\mathbf{r}|}+\sum_{i=1}^{N} \frac{z_{c}}{\left|\mathbf{r}_{i}-\mathbf{r}\right|}\right] \\
& \times \frac{e^{-\beta \mathcal{H}}}{\mathcal{Z}} d \mathbf{r}_{1} \cdots d \mathbf{r}_{N} .
\end{aligned}
$$

Since the counterion distribution $c(\mathbf{r})$ is related to the partition function by

$$
c\left(\mathbf{r}_{1}\right)=\frac{1}{(N-1) !} \int \cdots \int \frac{e^{-\beta \mathcal{H}}}{\mathcal{Z}} d \mathbf{r}_{2} \cdots d \mathbf{r}_{N},
$$

the electric potential can be rewritten as

$$
\beta z_{c} e \psi(\hat{\mathbf{r}})=-\frac{Z / z_{c}}{|\hat{\mathbf{r}}|}+\int_{V} \frac{c\left(\hat{\mathbf{r}}_{1}\right) \lambda^{3}}{\left|\hat{\mathbf{r}}_{1}-\hat{\mathbf{r}}\right|} d \hat{\mathbf{r}}_{1} .
$$

The above equation can also be written down directly from the Coulomb's law. When all counterions are condensed on the particle surface, one has the mean concentration $c(\mathbf{r})$ $=(N / V) \delta(|\mathbf{r}|-a)$ and hence the mean surface potential is zero, $\psi_{s}=0$.

In the WS cell model the osmotic pressure is given by ${ }^{12}$

$$
\pi=-\left(\frac{\partial \mathcal{F}}{\partial V}\right)_{N}=\frac{k_{B} T}{4 \pi R^{2}}\left(\frac{\partial \ln \mathcal{Z}}{\partial R}\right)=c(r=R) k_{B} T .
$$


Using Eqs. (8) and (10), one has

$$
\begin{aligned}
\ln c\left(\left|\mathbf{r}_{1}\right|=R\right) & \simeq\left[\frac{\beta \mathcal{F}(N)-\beta \mathcal{F}(N-1)}{1}\right]_{Z} \\
& \simeq\left(\frac{\partial \beta \mathcal{F}}{\partial N}\right)_{Z}=\beta \mu,
\end{aligned}
$$

where the equality is justified if the free energy of the system containing $N-1$ counterions is essentially equal to that containing $N$ counterions but with one counterion fixed at the cell surface, i.e., $\mathcal{F}(N-1) \simeq \mathcal{F}\left(N ;\left|\mathbf{r}_{1}\right|=R\right)$. Note that the particle charge $Z$ remains unchanged. This consequence indicates that the chemical potential can be obtained through the osmotic pressure measured in experiments. The methods of charge renormalization based on chemical potential and osmotic pressure are essentially equivalent.

Albeit the mean dipole associated to the system is zero due to spherical symmetry, the mean-square dipole moment can serve as an indicator of the charge renormalization. With the dipole moment defined as $\mathbf{P}=z_{c} \sum_{i=1}^{N} \mathbf{r}_{i}$, the mean-square dipole moment is given by

$$
\left\langle\mathbf{P}^{2}\right\rangle=\frac{1}{N !} \int \cdots \int \mathbf{P}^{2} \frac{e^{-\beta \mathcal{H}}}{\mathcal{Z}} d \mathbf{r}_{1} \cdots d \mathbf{r}_{N}=z_{c}^{2} \lambda^{2}\left\langle\sum_{i=1}^{N} \hat{\mathbf{r}}_{i}^{2}\right\rangle .
$$

When the thermal motion is dominant $\left\langle\mathbf{P}^{2}\right\rangle \sim O\left(N R^{2}\right)$. On the other hand, if all counterions are uniformly condensed on the particle surface, $\left\langle\mathbf{P}^{2}\right\rangle \sim O\left(N a^{2}\right)$. As the number of counterions is increased, $\left\langle\mathbf{P}^{2}\right\rangle$ may grow at first and decline later due to counterion condensation. The above equation also indicates that for the same set of $\{N, R / \lambda, a / \lambda\}$, one should arrive at the same result of $\left\langle\mathbf{P}^{2}\right\rangle / z_{c}^{2} \lambda^{2}$.

\section{A. Very weak coupling: Debye-Hückel approximation}

In general, the DH result of the thermodynamic quantity $\omega$ is used to perform charge renormalization. That is, $Z^{*}$ is the effective charge of a particle with intrinsic charge $Z$ when $\omega(Z)=\omega_{\mathrm{DH}}\left(Z^{*}\right)$. In the very weak coupling limit $\beta \mathcal{H} \ll 1$, one is able to obtain the DH results analytically from the partition function, Eqs. (1) and (2), by linearization. When $\beta \mathcal{H} \ll 1, \exp (-\beta \mathcal{H}) \simeq 1-\beta \mathcal{H}$ and the $N$-dimensional integration in the partition function becomes $N$ identical onedimensional integrals. Thereby the problem becomes tractable and the integral for a particular property can be carried out analytically.

When the thermal motion dominates, the internal energy $U$ can be evaluated analytically from the partition function,

$$
\begin{aligned}
\beta U & =\frac{1}{N !} \int \cdots \int \beta \mathcal{H} \frac{e^{-\beta \mathcal{H}}}{\mathcal{Z}} d \mathbf{r}_{1} \cdots d \mathbf{r}_{N}, \\
& \simeq-\Delta(N) \ll 1,
\end{aligned}
$$

where

$$
\begin{aligned}
\Delta(X)= & \frac{3}{2} X Z z_{c} l_{B} \frac{R^{2}-a^{2}}{R^{3}-a^{3}} \\
& -X(N-1) z_{c}^{2} l_{B} \frac{\frac{3}{5}\left(R^{5}-a^{5}\right)-\frac{3}{2} a^{3}\left(R^{2}-a^{2}\right)}{\left(R^{3}-a^{3}\right)^{2}} .
\end{aligned}
$$

Note that

$$
\mathcal{Z} \simeq \frac{V^{N}}{N !}[1+\Delta(N)]
$$

From Eq. (6), the configuration chemical potential is evaluated,

$$
\begin{aligned}
\beta \mu_{c} \simeq & -\frac{3}{2} Z z_{c} \ell_{B} \frac{R^{2}-a^{2}}{R^{3}-a^{3}} \\
& -2 N z_{c}^{2} \ell_{B} \frac{\frac{3}{5}\left(R^{5}-a^{5}\right)-\frac{3}{2} a^{3}\left(R^{2}-a^{2}\right)}{\left(R^{3}-a^{3}\right)^{2}} .
\end{aligned}
$$

Similarly, the thermal motion results in the counterion concentration uniform, as a first approximation, $c(r) \simeq N / V$, and thus Eq. (9) reduces to

$$
\begin{aligned}
\beta z_{c} e \psi= & -\frac{Z z_{c} \ell_{B}}{r}+\frac{3}{2} N z_{c}^{2} \ell_{B} \frac{R^{2}-\frac{1}{3} r^{2}-\frac{2}{3} \frac{a^{3}}{r}}{R^{3}-a^{3}} \\
= & \frac{4}{3} \pi \frac{\left[(\kappa R)^{3}-(\kappa a)^{3}\right]}{\kappa r}+2 \pi\left[(\kappa R)^{2}-\frac{1}{3}(\kappa r)^{2}\right. \\
& \left.-\frac{2}{3} \frac{(\kappa a)^{3}}{\kappa r}\right],
\end{aligned}
$$

where the inverse Debye length $\kappa$ is defined as $\kappa^{2}$ $=N / V z_{c}^{2} \ell_{B}$. Note that the electric potential satisfies the two boundary conditions for the PB equation: (1) $d \psi / d r=0$ at $r=R$ and (2) $\epsilon_{r} \epsilon_{0} d \psi / d r=Z / 4 \pi a^{2}$. That is, this result agrees with the solution of linearized PB equation with $\kappa r \ll 1$. The surface potential $\psi_{s}=\psi(r=a)$ is therefore

$$
\beta e \psi_{s}=-\frac{Z}{a} \ell_{B}\left[1-\frac{3}{2} \frac{a\left(R^{2}-a^{2}\right)}{R^{3}-a^{3}}\right] .
$$

The above expression clearly indicates that the DH surface potential is linearly proportional to $Z / a$ and the electric field established by the particle charge dominates the surface potential in a dilute dispersion $(R \gg a)$.

Performing the integration in Eq. (9) yields 


$$
\begin{aligned}
c(\mathbf{r})= & \frac{N}{V}\left\{1+\frac{Z z_{c} \ell_{B}}{r}-\frac{3}{2}(N-1) z_{c}^{2} \ell_{B}\right. \\
& \left.\times \frac{R^{2}-\frac{1}{3} r^{2}-\frac{2}{3} \frac{a^{3}}{r}}{R^{3}-a^{3}}-\Delta(1)\right\} \\
= & \frac{N}{V}\left[1-\beta e \psi\left(\mathbf{r} ; \mathbf{r}_{1}\right)-\Delta(1)\right] .
\end{aligned}
$$

Because $N / V$ does not represent the bulk concentration as in the simple liquid theory, the constant $\Delta(1)$ is present. Note that Eq. (17) does satisfy the conservation condition

$$
\int_{V} c(\mathbf{r}) d \mathbf{r}=N
$$

According to Eqs. (10) and (12), the osmotic pressure and mean-square dipole moment are given, respectively, by

$$
\pi=\frac{N}{V} k_{B} T\left[1+\frac{z_{c}^{2} \ell_{B}}{R}-\Delta(1)\right]
$$

and

$$
\left\langle\mathbf{P}^{2}\right\rangle=\frac{3}{5} N z_{c}^{6} \ell_{B}^{2} \frac{\hat{R}^{5}-\hat{a}^{5}}{\hat{R}^{3}-\hat{a}^{3}} .
$$

It is worth noting that all the aforementioned derivations are based on the partition function and do not resort to the PB equation.

\section{MONTE CARLO SIMULATION}

The condition of very weak coupling is justified only when $N^{2} z_{c}^{2} \ell_{B} / R \ll 1$. That is, it corresponds to low charged particle of large radius, low valency counterion, and high dielectric constant. However, this extremely stringent condition is often violated in most interesting situations. For strong electrostatic coupling, the partition function can only be calculated numerically. In order to take into account the effect of counterion fluctuation and correlation, we perform Monte Carlo simulations to evaluate the thermodynamic properties based on the WS cell model. In addition, the effect of dielectric contrast between the particle $\left(\epsilon_{p} \simeq 2\right)$ and the solvent $\left(\epsilon_{s} \simeq 80\right)$ is examined.

There are $N$ counterions with radius $d$ enclosed in a spherical cavity of radius $R$. The charged particle of radius $a$ is positioned at the center. These counterions are located at positions $\left\{\mathbf{r}_{i}\right\}$ with charges $z_{c} e$, where $i=1, \cdots, N$. The space charge distribution $a<\left|r_{i}\right|<R$ is related to the electric potential by the Poisson equation,

$$
\nabla \cdot\left[\epsilon_{r}(\mathbf{r}) \epsilon_{0} \nabla \psi(\mathbf{r})\right]=-\sum_{j=1}^{N} z_{c} e \delta\left(\mathbf{r}-\mathbf{r}_{j}\right) .
$$

Because of the linearity associated with Eq. (20), the electric potential is simply the sum of those caused by each ion. Inside the cavity, the solution of Eq. (20) due to the ion $k$ at $\mathbf{r}_{k}$ can be expressed analytically, ${ }^{15}$

$$
\psi_{k}(\mathbf{r})=-\frac{Z e}{4 \pi \epsilon_{s} \epsilon_{0} r}+\frac{z_{c} e}{4 \pi \epsilon_{s} \epsilon_{0}} \frac{1}{\left|\mathbf{r}-\mathbf{r}_{k}\right|}+\psi_{k}^{\prime}\left(r ; \frac{\epsilon_{p}}{\epsilon_{s}}\right) .
$$

$\psi_{k}^{\prime}(\mathbf{r})$ represents the image contribution due to dielectric differences and is given by

$$
\psi_{k}^{\prime}(\mathbf{r})=\frac{z_{c} e}{4 \pi \epsilon_{s} \epsilon_{0}} \sum_{i=0}^{\infty} B_{i}\left(\frac{a}{r}\right)^{i+1} P_{i}(\cos \theta)
$$

where $P_{j}$ is the Legendre polynomial and

$$
B_{i}=\frac{k\left(\epsilon_{s}-\epsilon_{p}\right)}{k \epsilon_{p}+(k+1) \epsilon_{s}} \frac{a^{k}}{r_{i}^{k+1}} .
$$

The angle $\theta$ is formed by the vectors $\mathbf{r}$ and $\mathbf{r}_{k}$ and $r_{k}=\left|\mathbf{r}_{k}\right|$. Note that the image contribution vanishes, $B_{i}=0$, if there is no dielectric mismatch, $\epsilon_{p}=\epsilon_{s}$. In the simulation, the infinite series of Legendre polynomials are truncated after the first 20 terms.

A brief description of MC simulations is given below. The simulation details can be seen elsewhere. ${ }^{15}$ The system simulated in this work consists of a collection of hard spheres. The valency of counterion is set to be $z_{c}=1,2$, or 3 . The diameter of the counterion is assumed to be $d$ $=0.4 \mathrm{~nm}$. We take $k_{B} T / e$ as the unit for the electric potential and $d$ for the spatial length. At $298 \mathrm{~K}$, the dimensionless energy parameter is given as $e^{2} /\left(4 \pi \epsilon_{s} \epsilon_{0}\right) k_{B} T d=1.785$. The simulations were performed under conditions of constant temperature, volume, and total number of counterions. The initial configuration for a given number of ions was obtained by randomly putting the ion within the cavity without overlapping each other. The system was equilibrated for about $10^{5} \mathrm{MC}$ steps per ion and the production period for each simulation was $5 \times 10^{5}$ steps per ion. The moves employed in our simulations were bead displacement motions. Bead displacement moves involve randomly picking a counterion and displacing it to a new position in the vicinity of the old position. The new configurations resulting from the moves were accepted according to the standard Metropolis acceptance criterion, $P_{\text {acc }}=\min \left[1, \exp \left(-\Delta U_{\mathrm{el}} / k_{B} T\right)\right]$, where $\Delta U_{\mathrm{el}}$ is the change in the total electrostatic energy of the system due to the move.

The chemical potential is given by ${ }^{16}$

$$
\mu=\mu_{0}+k_{B} T \ln c(r) d^{3}+\mu_{\mathrm{ex}}(r),
$$

where $\mu_{\mathrm{ex}}$ denotes the excess chemical potential. For convenience, the reference chemical potential is set to be zero, $\mu_{0}=0$. The ideal (counterion concentration) and excess chemical potentials vary with the radial position and can be evaluated from MC. We divide the spherical volume into 30 spherical shells and record the number of ions in each shell. The excess chemical potential is obtained by the Widom's method, ${ }^{16}$ which is the reversible work needed to add a counterion to the system,

$$
\mu_{\text {ex }}=-k_{B} T \ln \left\langle\exp \left(-\Delta U / k_{B} T\right)\right\rangle .
$$

Figure 1 shows typical distributions of counterion concentrations, excess chemical potential, and the total chemical potential. The counterion concentration $c(r)$ declines rapidly 


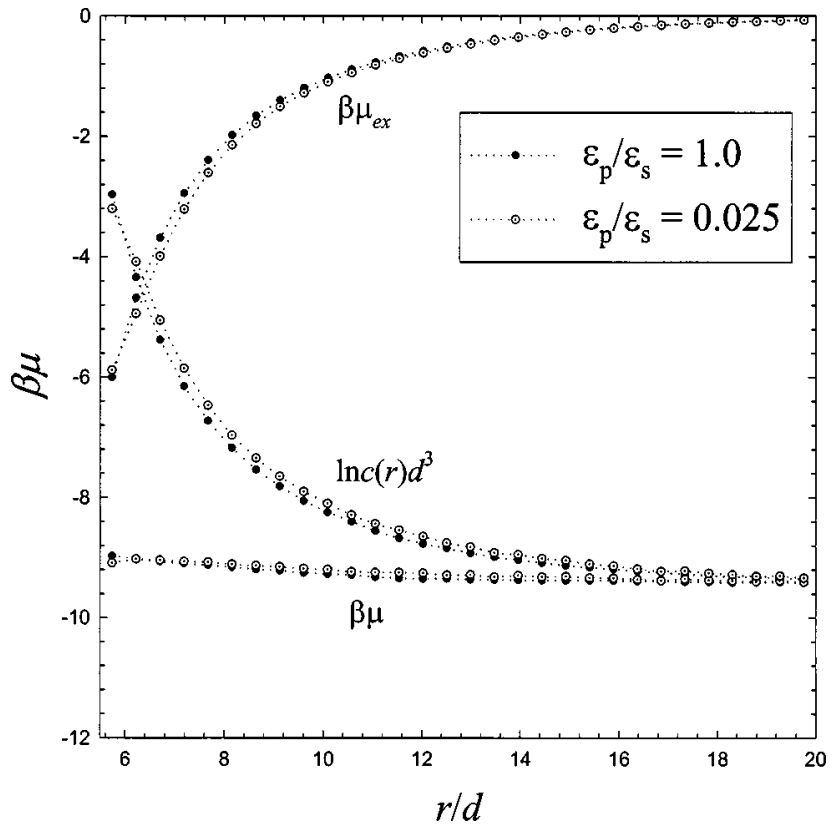

FIG. 1. The variation of the counterion concentration $\left(\ln c d^{3}\right)$, the excess chemical potential $\left(\mu_{\mathrm{ex}}\right)$, and the total chemical potential $(\mu)$ with the radial distance for $z_{c}=2, N=20, a=5 d$, and $R=20.5 d$. Two conditions are considered, dielectric continuity $\left(\epsilon_{p} / \epsilon_{s}=1.0\right)$ and dielectric discontinuity $\left(\epsilon_{p} / \epsilon_{s}=0.025\right)$. The dotted lines are drawn to guide the eyes.

from the particle surface to the boundary of the WS cell because of the attraction of the charged colloid. On the other hand, the excess chemical potential increases from $r=a+d$ and approaches zero at $r=R$ due to repulsion among counterions. The sum of $\ln c(r) d^{3}$ and $\mu_{\mathrm{ex}}(r)$ yield the total chemical potential, which is essentially constant as illustrated in Fig. 1. Note that the Widom's method may be inaccurate at small $N$ or concentrated regions. ${ }^{16}$

\section{RESULTS AND DISCUSSION}

When a salt-free colloidal dispersion contains colloidal particles with small radius and large amount of charges $Z$, the counterions and the particle are in strong electrostatic coupling. Under such a circumstance, the thermodynamic properties displayed by the dispersion may be the same as those associated with particle charge $Z^{*}$. The charge renormalization of intrinsic charge $Z$ by the effective charge $Z^{*}$ is commonly done based on the WS cell model. In this paper we investigate the charge renormalization of the intrinsic particle charge in accordance with the thermodynamic properties of the system, which are computed by MC simulations.

It is generally assumed that the thermodynamic behavior of the colloidal dispersion can be described by the mean-field theory, i.e., PB equation. As a result, the effective charge is determined by comparing the $\mathrm{DH}$ results to the $\mathrm{PB}$ results. That is, one can evaluate the effective charge based on the thermodynamic quantity $\omega$ with $\omega_{\mathrm{DH}}\left(Z^{*}\right)=\omega_{\mathrm{PB}}(Z)$. Obviously, in the weak coupling limit, one has $Z^{*}=Z$ because the $\mathrm{PB}$ result reduces to the $\mathrm{DH}$ result. When the electrostatic coupling is increased, the $\mathrm{PB}$ result deviates from the $\mathrm{DH}$ result. In general, one anticipates that the latter must be greater than the former if both are estimated based on the same intrinsic charge, i.e., $\omega_{\mathrm{DH}}(Z)>\omega_{\mathrm{PB}}(Z)>0$. If one adopts surface potential, osmotic pressure, or chemical potential as the interesting property, then the effective charge can be identified, respectively, by Eqs. (16), (18), or (14) with $Z^{*} \leqslant Z$.

Since the PB theory neglects ion fluctuations and correlations, significant deviations from the exact result based on the partition function are observed in strong electrostatic coupling regime. In order to accurately study the charge renormalization phenomenon associated with highly charged particles, we compute the thermodynamic properties numerically by MC simulations. We focus on the chemical potential (osmotic pressure) and surface potential. The former can be measured experimentally while the latter is closely related to the counterion distributions.

\section{A. Comparison between $\mathrm{DH}$ and $\mathrm{MC}$ results}

In the very weak coupling regime, the analytical expressions of chemical potential, surface potential, mean-square dipole moment, and internal energy can be obtained under the DH assumption. Figure 2(a) depicts the variation of the configuration chemical potential $\mu_{c}$ with the number of counterions $Z / z_{c}$. Under the same condition of $\{a / \lambda, R / \lambda\}$, data points of different combinations of $\left\{z_{c}, l_{B}, a, R,\right\}$ fall into a single curve. When $Z / z_{c}$ is small enough, the MC results are consistent with the DH theory, Eq. (14). Figure 2(b) illustrates the change of the total chemical potential $\mu$ with the intrinsic particle charge $Z / z_{c}$. Owing to the ideal chemical potential $\mu_{i}$, the total chemical potentials are separated into three curves even though they have the same $\{a / \lambda, R / \lambda\}$. Note that the configuration chemical potential grows with $N, \beta \mu_{c} \propto N$, more rapidly than the ideal chemical potential, $\mu_{i} \propto \ln N$. However, the proportional factor of $\mu_{c}$ is small and hence the ideal chemical potential dominates. The total chemical potential thus increases logarithmically with increasing $N$ in the very weak coupling regime.

Figures 2(c) and 2(d) plot the surface potential $\beta e \psi_{s}$ and the mean-square dipole moment $\left\langle\mathbf{P}^{2}\right\rangle$ against the number of counterions $N$ for different combinations of parameters, respectively. Again, all data points collapse into a single curve, which agrees with the DH expressions, Eqs. (16) and (19), at small $Z / z_{c}$. The variation of the internal energy with $Z / z_{c}$ is shown in Fig. 2(e). The internal energy can be separated into attraction between the particle and counterions and repulsion among counterions. One can clearly see that the attractive energy is always about twice larger than the repulsive energy. Both contributions can be well represented by the DH theory, Eq. (13). This result indicates that the internal energy always declines with increasing intrinsic particle charge, $\beta U \propto$ $-N^{2}$. In other words, the internal energy gain due to the attractive contribution may compensate the entropy loss as the intrinsic particle charge is increased. Overall, the excellent agreement between $\mathrm{DH}$ and $\mathrm{MC}$ results in the very weak coupling regime justifies both our theory and MC simulations. Evidently, the thermodynamic properties are essentially related to the intrinsic particle charge in this regime, instead of the effective charge. The analytical expressions associated with the DH theory are convenient for further use in theoretical and experimental studies. 

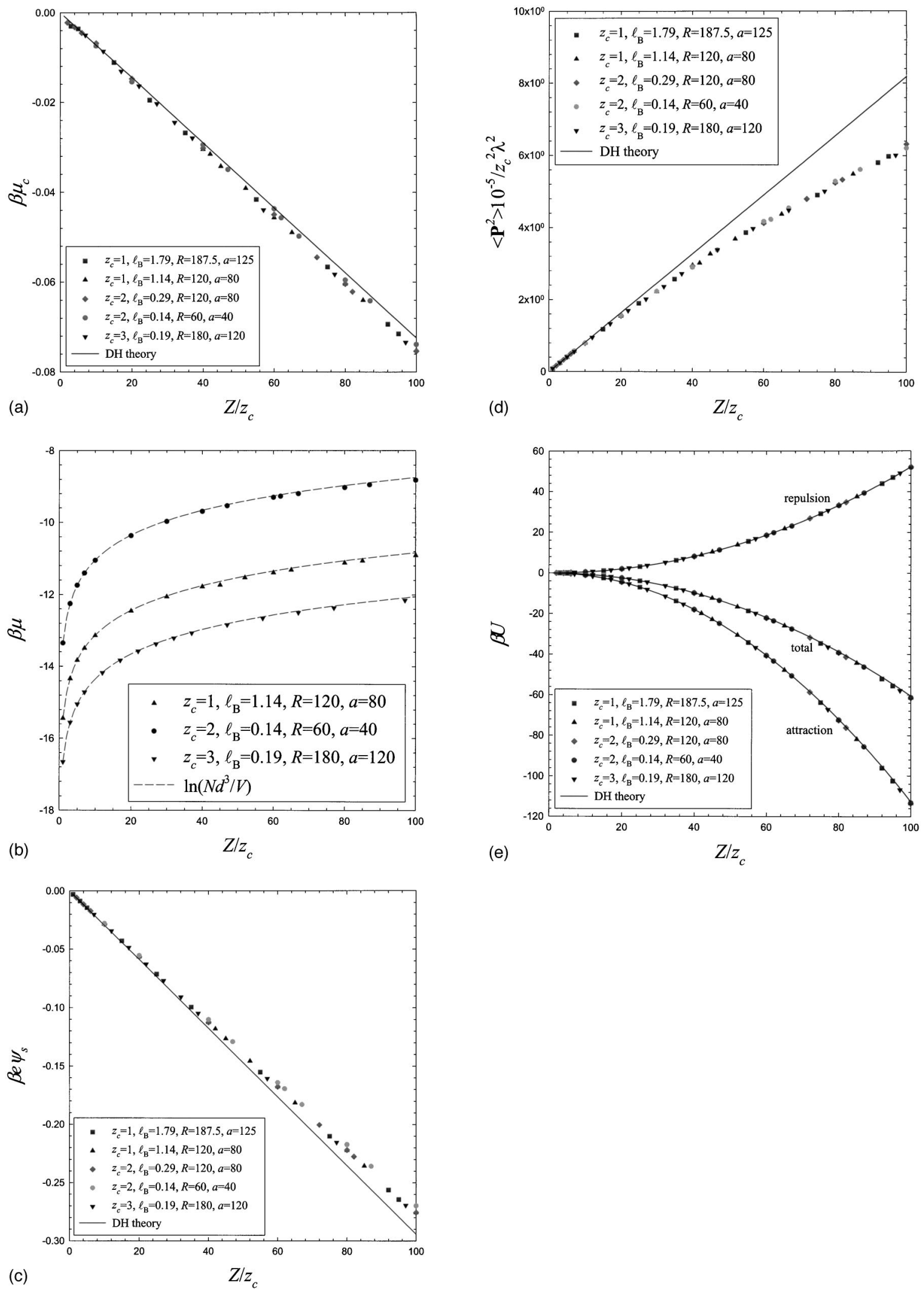

FIG. 2. (a) The configuration chemical potential is plotted against the number of counterions for various combination of parameters. (b) The variation of the total chemical potential with the number of counterions for different sets of parameters in the very weak coupling regime. (c) The electric potential on the particle surface is plotted against the number of counterions for different combination of parameters in the very weak coupling regime. (d) The mean-square dipole moment is plotted against the number of counterions for different combination of parameters in the very weak coupling regime. (e) The variation of the internal energy with the number of counterions in the very weak coupling regime. It includes the attraction between the particle and counterions and the repulsion among counterions. 


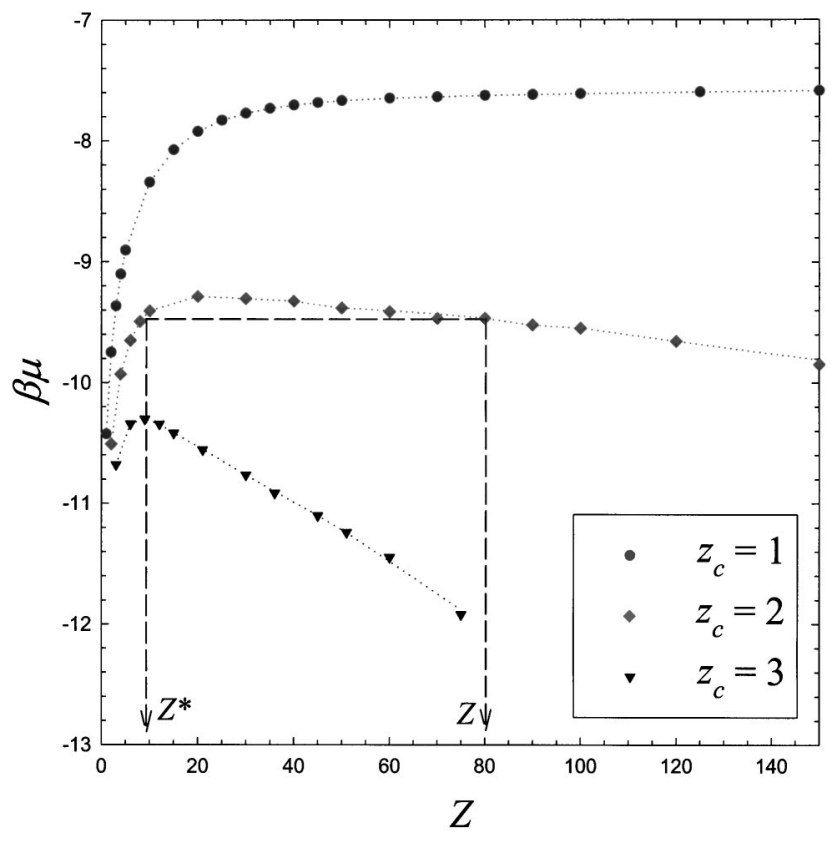

FIG. 3. The variation of the total chemical potential with the intrinsic particle charge for different counterion valency at $a / d=5$ and $R / d=20$.

\section{B. Charge renormalization by chemical potential (osmotic pressure)}

As indicated in Eq. (6), the total chemical potential $\mu$ consists of two parts, the ideal and configuration chemical potential, i.e., $\mu_{i}$ and $\mu_{c}$, respectively. The advantage of adopting the chemical potential as the basis for charge renormalization is that the chemical potential remains constant everywhere in the system. The osmotic pressure possesses the same characteristic and has been shown essentially equivalent to the chemical potential in Eqs. (10) and (11). It is very common to adopt the $\mathrm{DH}$ expression as the basis to determine the effective charge, i.e., $\mu_{\mathrm{DH}}\left(Z^{*}\right)=\mu(Z)$. Nonetheless, we shall show later that two dispersions with the same particle size and volume fraction but different intrinsic charges $\left(Z^{*}<Z\right)$ may display the same chemical potential, $\mu(\tilde{Z})=\mu(Z)$. Thereby, one can also define $\tilde{Z}$ as the effective charge.

We consider a typical example of charge renormalization, such as sodium counterion condensation on the surface of a SDS micelle. The ion diameter is $d=0.4 \mathrm{~nm}$ and the radius of the micelle is $a=2 \mathrm{~nm}$. If the surfactant concentration is $50 \mathrm{mM}$ with the aggregation number 80 , the radius of the WS cell is about $R=8 \mathrm{~nm}$. Figure 3 shows the variation of the chemical potential with the intrinsic particle charge $Z$ for different counterion valency at $a=5 d$ and $R=20 d$. For monovalent counterions, $z_{c}=1$, the chemical potential ascends fast at small $Z$ but seems to approach an asymptotic value for large intrinsic particle charge. In comparison with the $\mathrm{DH}$ chemical potential $\left(\simeq \ln Z^{*} d^{3} / z_{c} V\right)$, one is able to define the effective charge as $\mu_{\mathrm{DH}}\left(Z^{*}\right)=\mu(Z)$. When the intrinsic charge is large enough $(Z \gtrsim 50)$, the effective charge is essentially constant and independent of the intrinsic charge $\left(Z^{*} \simeq 16\right)$. This result seems to be in agreement with the PB theory, which predicts that the effective charge is increased with the intrinsic charge up to the point where the entropy balances the internal energy. From that point on, the effective charge remains constant. ${ }^{1,9}$

For divalent counterions $\left(z_{c}=2\right)$, however, we clearly observe that the chemical potential of counterions reaches a maximum value and declines with increasing $Z / z_{c}$. It is a consequence of the competition between the counterion entropy and the internal energy associated with the particlecounterion interactions. When the number of counterions is small, the entropy term, scaled as $\ln N$, dominates. On the other hand, the internal energy, scaled as $Z^{\delta}$ with $\delta>0$, becomes dominant as $Z$ is large. The maximum chemical potential corresponds to the point of equal importance for both contributions. The existence of a maximum $\left(Z=Z_{m}\right)$ also indicates that a dispersion with particle charge $Z>Z_{m}$ possesses the same chemical potential of counterions (osmotic pressure) as that with $Z^{*}<Z_{m}$. Note that both dispersions have the same characteristics of colloidal particles except intrinsic charges. As a consequence, one can define $Z^{*}$ as the effective charge for the intrinsic charge $Z$, as demonstrated by the arrows in Fig. 3. For trivalent counterions $\left(z_{c}=3\right)$, the maximum chemical potential takes place even earlier, i.e., at smaller intrinsic charge $Z_{m}\left(z_{c}=3\right)<Z_{m}\left(z_{c}=2\right)$. Therefore, the effective charge of trivalent counterions is smaller than that of divalent counterions for the same intrinsic charge $Z$. In view of the behavior associated with divalent and trivalent counterions, it is natural to anticipate that the chemical potential associated with monovalent counterions may eventually decline for large enough $Z$. In other words, in the strong coupling regime (very large $N$ for $z_{c}=1$ ), the PB theory fails and the ion fluctuations and correlations must be taken into account.

The analysis based on the partition function indicates that $\ln \left(1+\Omega_{N}\right)=f(N, R / \lambda, a / \lambda)$ and therefore the configuration chemical potential $\mu_{c}(N)$ of a given set $\{R / \lambda, a / \lambda\}$, should collapse into a single curve for different combination of $\left\{z_{c}, a, R, \ell_{B}\right\}$. Figure 4 confirms this result. Since $\lambda / R$ $\simeq 0.36$ does not satisfy the very weak coupling condition even for $N=1$ of those data points, the configuration chemical potential does not follow the DH result, $\mu_{c} \propto-N^{1}$. The effect of electrostatic coupling starts to contribute to the chemical potential $\mu_{c}$ and leads to the deviation, $\mu_{c} \propto-N^{\eta}$ with $\eta$ slightly greater than unity, for small number of counterions. However, as $N$ is increased, the growth of the configuration chemical potential with $N$ becomes slower and turn to $\mu_{c} \propto-N^{1 / 2}$. The exponent $1 / 2$ can be attributed to the strong correlation effect, which is ignored in the mean-field PB theory. In fact, the PB theory reveals that the configuration chemical potential behaves as $\mu_{c} \rightarrow-\ln N$ in the strong coupling regime. This is because the PB theory in the strong coupling limit assumes that

$$
\begin{aligned}
1+\Omega_{N} & =\frac{1}{\hat{V}^{N}} \int \cdots \int e^{-\beta \mathcal{H}} d \hat{\mathbf{r}}_{1} \cdots d \hat{\mathbf{r}}_{N} \\
& \sim\left[\frac{1}{\hat{V}} \int e^{-\beta z_{c} e \psi} d \hat{\mathbf{r}}\right]^{N} \simeq\left[\int c(r) d \hat{\mathbf{r}}\right]^{N}=N^{N} .
\end{aligned}
$$

As a result, the dependence of $\mu_{i}$ and $\mu_{c}$ on $N$ cancels out 


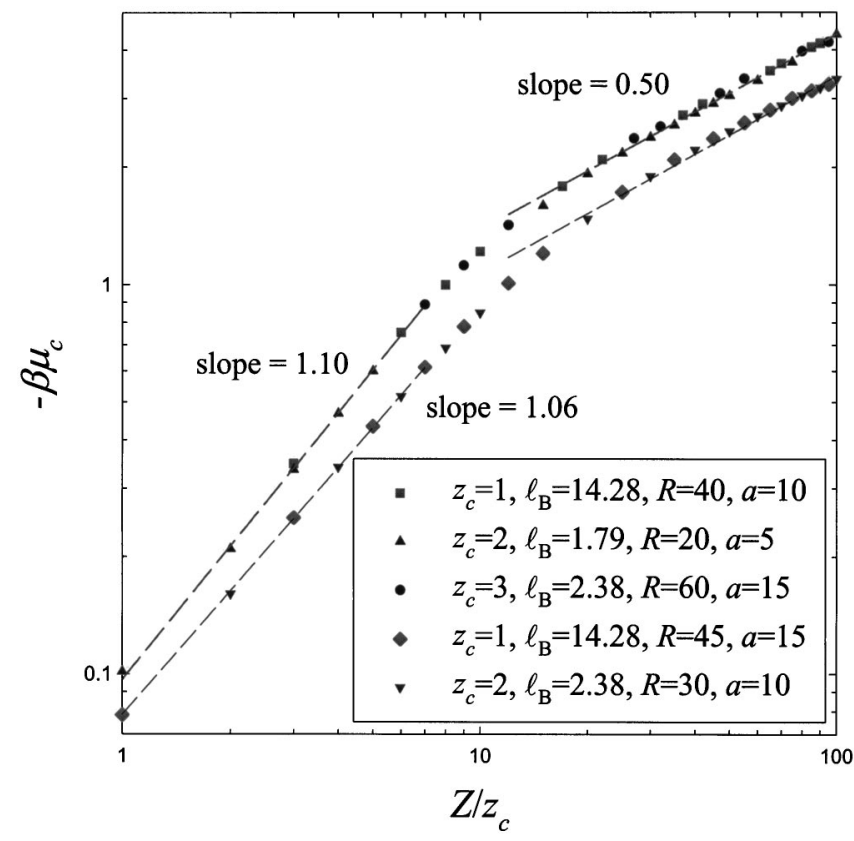

FIG. 4. The configuration chemical potential is plotted against the number of counterions for different combination of parameters in the strong coupling regime.

each and leads to an asymptotic values of both the total chemical potential $\mu\left(Z=Z_{m}\right)$ and the counterion concentration at the WS surface, $c\left(R ; Z=Z_{m}\right)$. For counterions condensed on a charged plane (two-dimensional system), the correlation effect of so-called strongly correlated liquid ${ }^{17}$ can be estimated based on the interaction energy of an ion with its WS cell of the background charge. This estimate gives $\mu \sim n^{1 / 2}$, where $n\left(=N / 4 \pi a^{2}\right)$ denotes the surface concentration. Albeit the counterions are not totally confined at the particle surface in the present study, the picture of strongly correlated liquid may shed some light on the behavior of the configuration chemical potential.

\section{Charge renormalization by surface potential}

The surface electric potential is also a good indication of charge renormalization. Figure 5 illustrates the variation of the surface potential with the intrinsic charge for different counterion valency with $a / d=5$ and $R / d=20$. Equation (9) shows that the surface potential $\psi_{s}=\psi(r=a)$ can be divided into two parts, the attractive and repulsive contributions. The attractive part is proportional to $N$ and is the dominant part. The repulsive part varies with the counterion distribution and reduces the absolute value of the surface potential. Two limiting conditions happen. When the thermal motion is dominant, the counterion concentration can be approximated to be uniform $[c(\mathbf{r}) \simeq N / V]$ and thereby one recovers the DH result, Eq. (16). On the other hand, if all counterions are accumulated near the surface, the repulsive contribution is essentially equal to the attractive part and therefore the surface potential approaches zero. As the electrostatic coupling is increased, the surface potential deviates from the thermal motion dominated regime $\left(\beta \psi_{s} \sim-Z \ell_{B} / a\right)$ toward the charge renormalization regime $\left(\beta \psi_{s} \rightarrow 0\right)$. The variation of

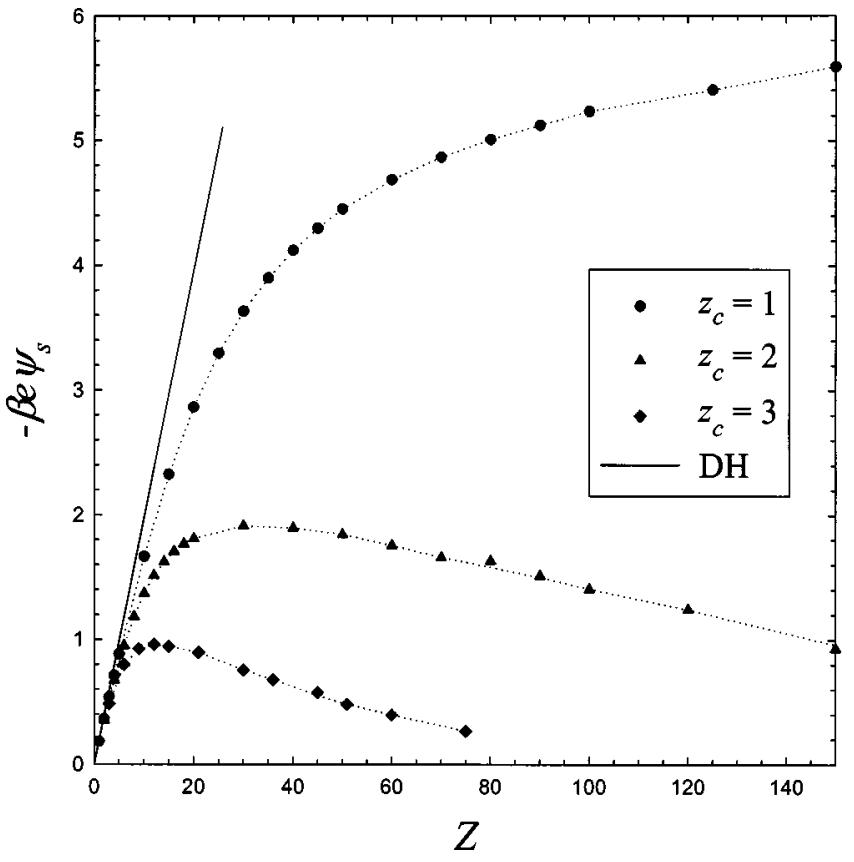

FIG. 5. The variation of the surface potential $\beta e \dot{\psi}(a+d / 2)$ with the intrinsic particle charge for different counterion valency at $a / d=5$ and $\dot{R} / d$ $=20$.

the surface potential with the intrinsic particle charge $\psi_{s}(Z)$ is able to demonstrate the extent of charge renormalization.

Similar to Fig. 3, one can define the effective charge by $\psi_{s}\left(Z^{*}\right)=\psi_{s}(Z)$ as well, particularly for multivalent counterions in Fig. 5. As to monovalent counterions, it is anticipated that the surface potential $-\beta e \psi_{s}$ will decrease ultimately at large enough $N$ and the above definition is appropriate. ${ }^{9}$ The renormalized charge can also be defined by the DH surface potential, Eq. (16). Since the DH expression, $-\psi_{s} \propto Z$, is always greater than that obtained from MC, i.e., $-\beta e \psi_{s, \mathrm{DH}}(Z)>-\beta e \psi_{s}(Z)$, the effective charge can be determined by $\psi_{s, \mathrm{DH}}\left(Z^{*}\right)=\psi_{s}(Z)$. To verify that monovalent counterions are able to display similar behavior as multivalent counterions, we examine the surface potential and the mean-square dipole moment of different valency but with the same $\{R / \lambda, a / \lambda\}$. Figures 6 and 7 show that all data points fall into a single curve and are consistent with the dimensionless forms of Eqs. (9) and (12). A maximum point of $-\beta z_{c} e \psi$ or $\left\langle\mathbf{P}^{2}\right\rangle / z_{c}^{2} \lambda^{2}$ is observed without doubt. Note that the surface potential calculated by the nonlinear PB equation is increased monotonically, and for large intrinsic charge the leading order behavior is

$$
\beta e \psi_{s} \sim-\ln Z^{2},
$$

which is qualitatively different from the MC results for multivalent counterions due to the neglect of the correlation effect in the strong electrostatic coupling regime.

For the charge renormalization phenomenon, the internal energy favors counterion accumulation on the surface but the entropy prefers counterions dispersed in the solvent medium. Figure 8 depicts the variation of the internal energy with the intrinsic charge. Similar to $\mu(\pi), \psi_{s}$, and $\left\langle\mathbf{P}^{2}\right\rangle$, the data sets associated with monovalent and trivalent counterions fall 


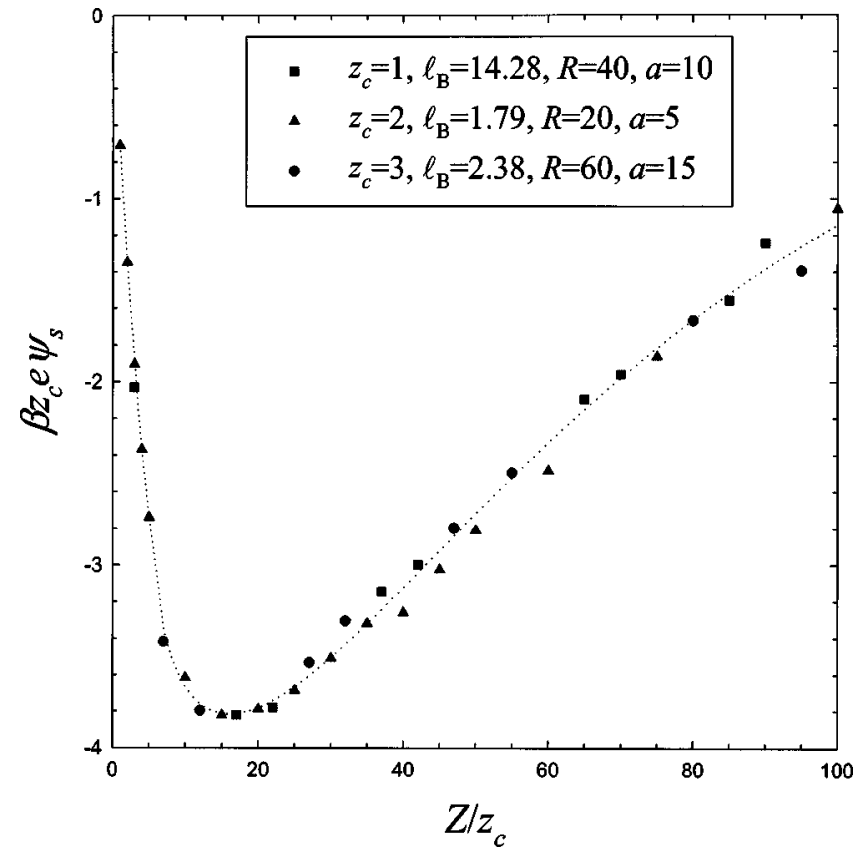

FIG. 6. The surface potential $\beta z_{c} e \psi(a+d / 2)$ is plotted against the number of counterions for different combination of parameters in the strong coupling regime. The dotted line is drawn to guide the eyes.

into the same curves because of the same $\{R / \lambda, a / \lambda\}$. The attractive contribution is about $U_{A} \approx-1.29 N^{2}$ while the repulsive one can be well fitted by $U_{R} \approx 0.54 N^{2}$. Thus, the total internal energy always decreases like $N^{2}$ as $Z$ is increased. Note that the absolute ratio of $U_{A}$ to $U_{R}$ decreases from $5 / 2$ of a dilute dispersion in the very weak coupling regime to 2 of totally condensed condition. In comparison to the mean-field contribution, the correlation effect is not significant. The internal energy gain $O\left(N^{2}\right)$ is able to compen-

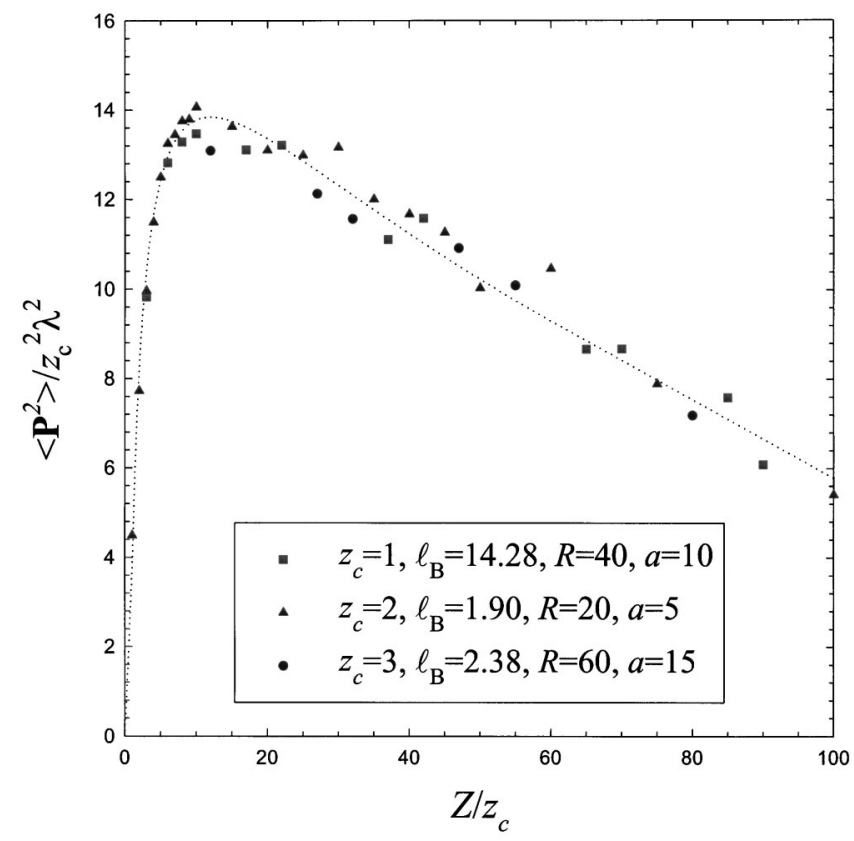

FIG. 7. The mean-square dipole moment is plotted against the number of counterions for different combination of parameters in the strong coupling regime. The dotted line is drawn to guide the eyes.

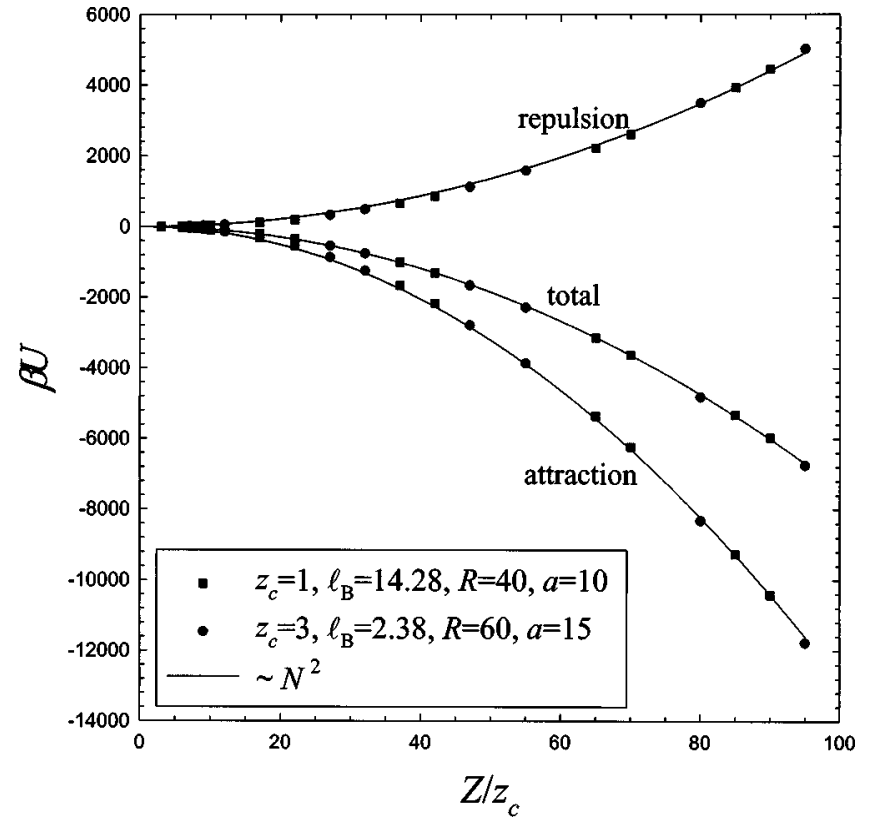

FIG. 8. The variation of the internal energy with the number of counterions in the strong coupling regime. It includes the attraction between the particle and counterions and the repulsion among counterions.

sate the entropy loss associated with counterions. In spite of the fact of the unimportant contribution associated with the correlation effect to the internal energy, its role is very crucial in determining the chemical potential. For simplicity, we consider the condition of total condensation. Based on the mean-field approximation, one has $\beta U_{A}=-Z N z_{c} \ell_{B} / a$ and $\beta U_{R}=1 / 2 N z_{c}^{2} \ell_{B} / a$. The internal energy part of the chemical potential is therefore $(\partial U / \partial N)_{z}=0$. The chemical potential is calculated by inserting one more counterion with the intrinsic charge $Z$ held fixed. Thus the correlation contribution must come into play and balances the change in the entropy part of the chemical potential, i.e., nonuniform counterion distribution.

\section{Effect of dielectric discontinuity}

In general, the charged colloid possesses typically a low dielectric constant (about 2-5), which is small compared to the surrounding solvent (about 80 for water). In most of the theoretical works, however, the dielectric contrast is neglected. In addition to Coulomb interactions, there is another contribution stemming from the dielectric discontinuity, i.e., the image force..$^{15}$ That is, at the particle-solvent interface, surface charges are induced owing to electric polarization. Thereby, the charge positioned in the solvent with higher dielectric constant feels an electrostatic repulsion from an "image charge" (a continuous linear charge distribution located in the colloidal particle) whose net charge has the same sign. ${ }^{18}$

The effects of image charges in spherical geometry have been studied recently. ${ }^{18}$ Convergence of the Legendre sums with a relative error of $10^{-6}$ was obtained by keeping the first 100 terms. ${ }^{18}$ It is found that the effect of image forces declines with increasing the number of counterions. ${ }^{18}$ Moreover, for multivalent counterions, ${ }^{18}$ a maximum counterion 
TABLE I. Effects of dielectric discontinuity with $z_{c}=2$. A comparison of chemical potential and surface potential with/without dielectric contrast.

\begin{tabular}{rcccc}
\hline \hline \multirow{2}{*}{$Z$} & $\begin{array}{c}\beta \mu\left(\epsilon_{p} /\right. \\
\left.\epsilon_{s}=0.025\right)\end{array}$ & $\begin{array}{c}\beta \mu\left(\epsilon_{p} /\right. \\
\left.\epsilon_{s}=1\right)\end{array}$ & $\begin{array}{c}\beta e \psi_{s}\left(\epsilon_{p} /\right. \\
\left.\epsilon_{s}=0.025\right)\end{array}$ & $\begin{array}{c}\beta e \psi_{s}\left(\epsilon_{p} /\right. \\
\left.\epsilon_{s}=1\right)\end{array}$ \\
\hline 20 & -9.22 & -9.27 & -4.12 & -3.62 \\
40 & -9.29 & -9.33 & -4.30 & -3.79 \\
80 & -9.47 & -9.46 & -3.35 & -3.26 \\
120 & -9.61 & -9.65 & -2.60 & -2.49 \\
150 & -9.90 & -9.81 & -1.88 & -1.86 \\
200 & -10.15 & -10.18 & -1.29 & -1.06 \\
\hline \hline
\end{tabular}

concentration is located very close to the particle surface, i.e., $[r-(a+d / 2)] / d<0.5$. In our simulations, the first 20 terms were kept in order to save the computing time. The typical error of the energy calculation is less than $1 \%$ and thus accurate enough results can be obtained in our simulations. Since we focus on the chemical potential distributed in the system, only 30 spherical shells are used to collect the data. The width of the interval is too large and therefore we are unable to see the maximum point in $c(r)$ as reported in Ref. 18.

We examine the effect of dielectric discontinuity by comparing the MC results of the chemical potential and surface potential with/without the image contribution. Figure 1 shows that the distributions of counterions and the excess chemical potential for $z_{c}=2, Z=40, a=5 d$, and $R=20 d$. In the neighborhood of the surface of the charged particle, the counterion concentration of $\epsilon_{p} / \epsilon_{s}=1$ is higher than that of $\epsilon_{p} / \epsilon_{s}=0.025$ due to polarization charges. However, the excess chemical potential with $\epsilon_{p} / \epsilon_{s}=1$ is larger than that of $\epsilon_{p} / \epsilon_{s}=0.025$. As a result, the total chemical potential of both cases are essentially the same. Table I lists both total chemical potential and surface potential as a function of the particle charges. The results further indicate that the influence of dielectric discontinuity is not significant in evaluating the chemical potential. Nonetheless, the surface potential is significantly affected by the concentration profile of counterions and thus $|\beta e \psi|$ with dielectric contrast is larger than that with $\epsilon_{p} / \epsilon_{s}=1$ because counterions are pushed away from the interface for the former case.

In the strong coupling regime, the decline of the chemical potential, surface potential, or mean-square dipole moment with increasing $N$ is attributed to the correlation effect, i.e., mutual ordering of counterions near the particle surface. ${ }^{9}$ For two colloidal dispersions containing charged particles of the same size and volume fraction, the particular thermodynamic property $(\omega)$ of them may be the same even though they possess different particle charges $Z^{*}$ and $Z$, i.e.,
$\omega\left(Z^{*}\right)=\omega(Z)$. As a consequence, one may define $Z^{*}$ as the effective charge for the dispersion with $Z$ if $Z>Z^{*}$. Another common approach for the effective charge determination is the DH expression, which is valid under the condition of very weak coupling, that is, $\omega_{\mathrm{DH}}\left(Z^{*}\right)=\omega(Z)$. The advantage of the latter method is that analytical expressions are generally available for various properties and further uses. Compared to the surface potential, the chemical potential or osmotic pressure remains constant everywhere in the system and is relatively insensitive to the dielectric discontinuity. The aforementioned approaches can be directly applied to salty dispersions in determining the effective charge. In the present cell simulations, the effect of colloid-colloid interaction is not taken into account because neighboring particles were absent. Our approach is justified for dilute dispersions. In addition, previous study ${ }^{9}$ also concluded that the results based on WS cell simulations agree reasonably well with those obtained by periodic boundary condition.

\section{ACKNOWLEDGMENTS}

This research was supported by National Council of Science of Taiwan under Grant No. NSC-92-2214-E-008-001. Computing time provided by the National Center for HighPerformance Computing of Taiwan is gratefully acknowledged.

${ }^{1}$ S. Alexander, P. M. Chaikin, P. Grant, G. J. Morales, and P. Pincus, J. Chem. Phys. 80, 5776 (1984).

${ }^{2}$ E. Trizac, L. Bocquet, and M. Aubouy, Phys. Rev. Lett. 89, 248301 (2002).

${ }^{3}$ J. M. Roberts, J. J. O'Dea, and J. G. Osteryoung, Anal. Chem. 70, 3667 (1998).

${ }^{4}$ G. S. Manning, J. Chem. Phys. 51, 924 (1969).

${ }^{5}$ F. Oosawa, Polyelectrolytes (Dekker, New York, 1971).

${ }^{6}$ B. H. Zimm and M. Le Bret, J. Biomol. Struct. Dyn. 1, 461 (1983).

${ }^{7}$ W. M. Gelbart, R. F. Bruinsma, P. A. Pincus, and V. A. Parsegian, Phys. Today 53, 38 (2000).

${ }^{8}$ M. J. Stevens, M. L. Falk, and M. O. Robbins, J. Chem. Phys. 104, 5209 (1996).

${ }^{9}$ R. D. Groot, J. Chem. Phys. 95, 9191 (1991).

${ }^{10}$ G. V. Ramanathan, J. Chem. Phys. 88, 3887 (1988).

${ }^{11}$ L. Belloni, Colloids Surf., A 140, 227 (1998).

${ }^{12}$ M. Deserno and C. Holm, Electrostatic Effects in Soft Matter and Biophyiscs (Kluwer Academic, Netherlands, 2001), p. 27; H. Wennerström, B. Jönsson, and P. Linse, J. Chem. Phys. 76, 4665 (1982).

${ }^{13}$ V. Sanghiran and K. S. Schmitz, Langmuir 16, 7566 (2000).

${ }^{14}$ E. J. W. Verwey and J. T. G. Overbeek, Theory of the Stability of Lyophobic Colloids (Elsevier, Amsterdam, 1948).

${ }^{15}$ Y.-J. Sheng and H.-K. Tsao, Phys. Rev. Lett. 87, 185501 (2001); Y.-J. Sheng and H.-K. Tsao, Phys. Rev. E 66, 040201(R) (2002); C.-H. Ho, H.-K. Tsao, and Y.-J. Sheng, J. Chem. Phys. 119, 2369 (2003).

${ }^{16}$ D. Frenkel and B. Smit, Understanding Molecular Simulation (Academic, New York, 1996).

${ }^{17}$ B. I. Shklovkii, Phys. Rev. E 60, 5802 (1999).

${ }^{18}$ R. Messina, J. Chem. Phys. 117, 11062 (2002). 